Int. J. Electrochem. Sci., 14 (2019) $9921-9935$

\title{
Characteristics and Microbial Activity of Shewanella and Escherichia coli under a Direct-Current Electric Field
}

\author{
Chen Dou ${ }^{1}$, Yiyang Liu ${ }^{1}$, Di Yin ${ }^{1}$, Yang Jiao ${ }^{1}$, Jiali Xu' ${ }^{1}$, Guangtuan Huang ${ }^{1}$, Lehua Zhang ${ }^{1,2, *}$ \\ ${ }^{1}$ State Environmental Protection Key Laboratory of Environmental Risk Assessment and Control on \\ Chemical Process, School of Resources and Environmental Engineering, East China University of \\ Science and Technology, Shanghai 200237, P. R. China. \\ ${ }^{2}$ Shanghai Institute of Pollution Control and Ecological Security, Shanghai 200092, P. R. China. \\ *E-mail: lezhanghua@163.com
}

doi: $10.20964 / 2019.10 .48$

Received: 9 July 2019 / Accepted: 17 August 2019 / Published: 30 August 2019

The effects of a direct-current electric field on cell viability and physicochemical properties of exoelectrogens and non-exoelectrogens, e.g., Shewanella and Escherichia coli, were studied. The results obtained through the analysis of differences in growth patterns and growth rates for Shewanella and Escherichia coli under electric fields with varying intensity showed that the direct-current electric field accelerated the growth of microbes. More Escherichia coli grew in the cathode, and more shewanella grew in the anode. When Shewanella and Escherichia coli were used as the culture, the activation resistance accounted for a large proportion of the total resistance, and the activation resistance with Shewanella was much less than that with Escherichia coli. The anode capacitance with Shewanella was higher than that with Escherichia coli, indicating that the anode capacitance is negatively correlated with the resistance.

Keywords: Electrochemistry analysis; Shewanella; Escherichia coli; Direct-current electric field; Bioelectrochemical system

\section{$\underline{\text { FULL TEXT }}$}

(C) 2019 The Authors. Published by ESG (www.electrochemsci.org). This article is an open access article distributed under the terms and conditions of the Creative Commons Attribution license (http://creativecommons.org/licenses/by/4.0/). 